\title{
Range and distribution effects on number line placement
}

\author{
Simon Kemp ${ }^{1} \cdot$ Matt Grice $^{1} \cdot$ Dena Makarious $^{1} \cdot$ Kate Stuart $^{1} \cdot$ Georgina C. Carvell $^{1} \cdot$ Nicola J. Morton $^{1}$. \\ Randolph C. Grace ${ }^{1}$
}

Accepted: 17 November 2020 / Published online: 6 January 2021

(C) The Psychonomic Society, Inc. 2021

\begin{abstract}
People's placement of numbers on number lines sometimes shows linear and sometimes compressive scaling. We investigated whether people's placement of numbers was affected by their range and distribution, as indicated by Parducci's (Psychological Review, 72, 407-418, 1965) range-frequency theory. Experiment 1 found large compressive effects when the endpoints were 1 and $10^{16}$. Experiment 2 showed compression when 14 logarithmically distributed numbers were placed on a line marked 1-1,000 and close to linear scaling when the numbers were linearly distributed. Thus, we found both range and frequency effects on compression. Where compression arose, it was not as pronounced as that predicted by logarithmic scaling, but analyses of the results from Experiments 1 and 2 indicate this was not explained by participants switching between linear and logarithmic scaling.
\end{abstract}

Keywords Compressive scaling $\cdot$ Number lines $\cdot$ Range-frequency theory

\section{Introduction}

When young children are asked to indicate the appropriate placement of numbers on a line that is bounded below by 0 and at the upper end by (say) 10,20 or 100, their placements are likely to display non-linearity and compression. A disproportionate section of the line may be allotted to the smaller numbers, and the physical distance between 2 and 4 will often be greater than the difference between 7 and 9 (e.g. Dehaene, 1997; de Hevia \& Spelke, 2009; Droit-Volet, Clément, \& Fayol, 2008; Siegler, 2009; Siegler \& Booth, 2004). This phenomenon has sometimes been taken as suggesting that children simply cannot represent numbers in the way adults do, but other interpretations are also possible (e.g. Cohen \& Sarnecka, 2014; Moeller, Pixner, Kaufmann, \& Nuerk, 2009; Slusser \& Barth, 2017; Zax, Slusser, \& Barth, 2019).

Although adults often make linear placement of numbers on lines, they too sometimes demonstrate compression (e.g. Cohen \& Blanc-Goldhammer, 2011; Dehaene, Izard, Spelke, \& Pica, 2008; Dotan \& Dehaene, 2016; Huber, Moeller, \& Nuerk, 2014; Izard \& Dehaene, 2008). Moreover, a number of

Simon Kemp

Simon.Kemp@canterbury.ac.nz

1 Psychology Department, University of Canterbury, Private Bag 4800, Christchurch 8140, New Zealand the adult findings, as well as some of those obtained from children, indicate that the extent and nature of compression depends on the experimental context and what training or instruction the participants are given (e.g. Cohen \& BlancGoldhammer, 2011; Göbel, Shaki, \& Fischer, 2011; Huber et al., 2014; Izard \& Dehaene, 2008). One way to interpret these results is to consider that humans may not have a single innate number line (Núñez, 2011) and that adults at least are quite capable of a number of different ways of representing or producing magnitudes.

Support for our possession of a range of different magnitude estimation capabilities can be found outside of number line research. Natural addition is the minimum of all monotonic functions of the natural numbers. Thus, if humans are responsive to monotonicity (functions always increase), addition of whole numbers is, in a sense, the simplest operation to perform on them (Grice, Kemp, Morton, \& Grace, 2020). Indeed, such additive systems are widely used in everyday life. Consider estimating the number of paving stones to use on a narrow path or calculating the right amount of change to give after being presented with notes of different denominations.

Psychophysics provides many examples of compressive estimation of sensory magnitudes. As a single example, consider how we respond to sounds of different levels. The range of sound intensity from the faintest sound that we can hear to that of the loudest sound we can hear without immediate hearing damage is about $1-10^{15}$ (e.g. Moore, 2004, Table 1.1). Because of this colossal range, we frequently express sound 
levels in terms of the logarithmic decibel scale $\left(10 \log _{10}\left(\mathrm{I} / \mathrm{I}_{0}\right)\right.$, where I stands for the intensity level and $\mathrm{I}_{0}$ is a reference intensity level). Weber's Law holds well for wideband noise, and the difference threshold intensity in decibels is the same (about $0.5-1 \mathrm{~dB}$ ) for noise regardless of the sound level (Moore, 2004, pp. 138-139). If we were to regard each difference threshold as a single unit of psychological sensation, there would then be a logarithmic relationship between psychological sensation and sound intensity, as Fechner pointed out long ago (Stevens, 1961).

Compression in sensory dimensions does not have to imply logarithmic scaling. Another way to compress sound intensities, the sone scale, uses a power-law function (Stevens, 1961, 1975). To sum up, many features of the way we respond to sound intensity, as well as to many other sensory dimensions, are explained by assuming that we compress the range.

Both power-law and logarithmic functions are reasonably common in psychophysics. Indeed, on occasion, the same modality can display either a power-law or logarithmic relationship depending on the way participants are instructed to estimate the modality. Magnitude estimation tends to produce power-law relationships; category rating tends to produce logarithmic relationships (e.g. Galanter, 1962a; Kemp, 1991).

There is also evidence that people placing numbers on lines can do so in different ways. Huber et al. (2014) found that adults could be readily trained to make non-linear placements. Grace, Morton, Ward, Wilson, and Kemp (2018) presented pairs of stimuli and required clicking on a line as a response. They found simple right/wrong feedback effective in steering participants towards either ratio or difference judgements.

The experiments in the present paper aimed to see if Parducci's (1965) range-frequency theory could explain some features of how adults assign numbers to number lines. Range-frequency theory, which can be classed as a psychophysical theory, explains how people set up categories for rating judgements of different stimuli along some dimension, and suggests that, when people set up boundaries between categories, two features of the stimuli are taken into account. First, the range between the greatest and smallest stimulus, and, second, the frequency distribution of the stimuli. In the original model the boundaries between a seven-category system are formed by taking the average of the boundaries indicated by the range ((greatest stimulus - smallest stimulus)/7) and the boundaries indicated by the frequency distribution (a seventh of the stimuli would fall into each category).

There is reasonable evidence for range-frequency theory (e.g. Parducci, 1965, 1974; Parducci \& Wedell, 1986). Application of the theory to the placement of numbers on number lines is suggested, in a general sense, because rangefrequency theory is an account of how context affects measurement, and placement of numbers on lines is affected by context (e.g. Barth \& Paladino, 2010; Huber et al., 2014). A consequence of range-frequency theory is that, as a rule, category boundaries will not be linearly placed, and, if the distribution of stimuli is positively skewed, as our everyday use of numbers is, the boundaries will show compression. Range-frequency theory resembles the approach of Cicchini, Anobile, and Burr (2014) to number placement in seeking to explain non-linearity from the effects of previously presented stimuli rather than from assuming a non-linear mental representation of number.

There are theoretical difficulties in trying to explain number line placements using the range-frequency theory as originally proposed by Parducci (1965). Most obviously, when numbers are placed on lines, they are not simply put into categories, and often participants are careful to assign a slightly lower number than a previous one to a position that is slightly to its left. Nonetheless, application of the principles of range-frequency theory to number placement does make two clear predictions.

Firstly, the amount of compression shown in placements on number lines should depend on the range of the stimuli and endpoints of the number line. Such findings have already been reported, although they have been explained in terms of number words (e.g. hundreds, millions) rather than rangefrequency theory (Landy, Charlesworth, \& Ottmar, 2017; Landy, Silbert, \& Goldin, 2013). We examined the effect of range in Experiment 1 below, using numerical stimuli that were not easy to express as words.

Secondly, given a constant range, the amount of compression should depend on the frequency distribution of the numbers. To our knowledge this prediction has not been previously investigated systematically by researchers, and it is examined in Experiment 2.

Given that compression is obtained, what form might it take? Some researchers (e.g. Dotan \& Dehaene, 2016) have suggested that number-line compression follows a logarithmic function. However, there is also reasonable evidence for segmented functions in which smaller numbers are fitted by a linear function and larger ones by a different function, sometimes another linear one with a shallower slope (e.g. Landy et al., 2013; Moeller et al., 2009). It is possible that segmented functions are indicated, perhaps necessitated, by the common, although not universal (Cohen \& Blanc-Goldhammer, 2011; Landy et al., 2013, 2017), use of zero as the lower marker on the number line. This use creates a contradiction for any participant wishing to use a true logarithmic function. The logarithm (to any base) of zero is - $-\infty$ (or does not exist), and strictly speaking it is impossible to include zero on any logarithmically scaled number line. Thus, if there is a lower marker of zero it cannot be a point on such a line. (For discussion of the implications of including zero on number lines for compressive scaling, see Didino et al., 2019.)

In Experiment 1 we biased the stimuli towards logarithmic placement by using logarithmically distributed stimuli. A consequence of our bias was the frequent presentation of decimal 
rather than whole numbers. In both experiments we chose a lower number line marker of 1 rather than 0 (the log to any base of 1 is zero). We did not expect that these experimental conditions would necessarily produce logarithmic functions. As outlined above, a power law provides a reasonable functional alternative to a logarithmic function. Moreover, if the form of compression is determined contextually, as is indicated by range-frequency theory, we should not expect any clear functional solution. However, one important aim in both our experiments was to see if logarithmic placement might come about in circumstances where it was mathematically reasonable for this to occur.

\section{Experiment 1}

This experiment varied the range of numbers to be placed and the line markers. In all three conditions the numbers to be placed were logarithmically identically spaced in terms of line distance, and used a lower marker of 1. (All logarithmic calculation here and below used base 10.) Three higher markers were used: 10,1000 and $10000000000000000\left(10^{16}\right)$. In one condition, subjects placed numbers on lines; in the other condition the numbers were denoted as dollars and participants were asked to assess value. Previous research suggested perceived value (or utility) of monetary sums is a compressive function of the monetary value, so we thought the value conditions might be more likely to produce compression than the plain numbers (Galanter, 1962b).

This experiment was preceded by an unreported study with more restricted marker ranges (upper markers 10 and 1,000) that found rather small range effects, but did establish that participants were capable of using a lower marker of 1 appropriately.

\section{Method}

Sixty participants (24 male; 26 female; median age 24 years, range 16-84; no age or gender information for ten participants) completed a questionnaire. Thirty-five completed number-line questionnaires and 25 completed value-line questionnaires. Participants were recruited casually by seven paid research assistants, with number- and value-line questionnaires randomly assigned.

Each participant placed four numbers or values on each of three lines (12 placements in total), with one line per landscape-oriented page. (The Appendix shows how one of these pages appeared to participants, and may be useful to the reader in interpreting the method.) All lines extended for 245 $\mathrm{mm}$, with the lower marker placed at $60 \mathrm{~mm}$ and the higher marker at $220 \mathrm{~mm}$. The lower mark was always 1 . The three high markers were 10, 1000 and 10000000000000000000 (no commas inserted). Numbers (or values) to be placed were:
High marker 10: 0.56, 1.15, 2.37, 4.87

High marker 1000: 0.18, 1.54, 13.3, 115.5

High marker 10000000000000000: 0.0001, 10, 1000000, 100000000000 .

These numbers were presented under the number line in a single randomly determined order (i.e. not sequentially).

In the value-line questionnaires all numbers, including the markers, were preceded by the symbol $\$$. The numbers were chosen to provide equal spacing on a logarithmic scale, and the four numbers (or values) to be placed would be at 20, 70, 120 and $170 \mathrm{~mm}$ from the extreme left hand of the line if logarithmic scaling were used. Actual placements were also recorded in $\mathrm{mm}$ from the extreme left-hand of the line. The pagination of the three different lines was randomly varied.

Instructions for the number and value lines were, respectively:

"Above is a number line. Below is a list of four numbers. Please indicate how you would like to place each number on the line by writing it near the line with a little arrow to show where it best fits in on the line. Please place the numbers where you think looks good to you. You can place the numbers anywhere on the line, including outside of the arrows."

"Above is a value line. Below is a list of four amounts of money. Please indicate how you would like to place each amount of money on the line by writing it near the line with a little arrow to show where it best fits in on the line. Please place the money amounts wherever you think best suits the value to people of that amount of money. You can place the money amounts anywhere on the line, including outside of the arrows."

For 11 of the number-line questionnaires, after the participants had completed their number placements, they were instructed:

"The following questions ask about your use of number words (e.g. a hundred, fifty-five, ten thousand, point five) when making your decisions. You are asked separately for each number list. (It might help to turn back to remind yourself of what you did.) To what extent did you use number words to help you place the numbers?"

The three lists were each responded to with "Not at all", "For some numbers" or "For all the numbers".

\section{Results}

Figures 1, 2, and 3 show the mean placement values alongside those expected from logarithmic and linear placement for each of the three high markers. All measures were taken from the extreme left of the line (not the lower marker). Clearly the 
mean values were quite close to the linear expectations when the High marker was 10, but veered towards logarithmic placement for the 1000 High marker and veered more still for the High marker of $10^{16}$. Analysis of variance of placement showed significant main effects of the marker, $F(2,116)$ $=38.6, p<.0001$, partial $\eta^{2}=.40$, the number (perhaps best thought of as logarithmic placement; $F(3,174)=363.1, p<$ .0001 , partial $\eta^{2}=.86$ ), and most importantly the interactive effect of these two, $F(6,348)=39.3, p<.0001$, partial $\eta^{2}=$ .40. However there was no significant effect of setting the task as a number or value line $\left(F(1,58)=.52, p=.47, \mathrm{MS}_{\text {Error }}=\right.$ $731)$ and no significant $(p<.05)$ interactive effects of this factor with the other two. Hence, the rest of this section merges results from the number- and value-placement tasks.

The results clearly show that the higher markers produce a tendency towards logarithmic scaling. However, examination of Figs. 2 and 3 shows that for at least the two highest numbers to be placed, the mean results are somewhere between those expected from logarithmic and linear scaling. Does this come about because some participants adopted logarithmic scaling for these numbers and some adopted linear scaling, and thus there was switching from one type of scale to the other? Alternatively, it may be that individual participants adopted a compromise placement that was neither linear nor logarithmic scaling.

The issue can be investigated from the distribution of the placement values for the two highest numbers for the two higher markers. If people were either using a linear scale or a logarithmic scale but switched between them, we would expect the distributions to be bimodal, with the modes centring on, for example, $62 \mathrm{~mm}$ and $120 \mathrm{~mm}$ for placing 13.3 on the 1000
High marker line. Of course, we could not expect all the linear responses to be placed exactly at $62 \mathrm{~mm}$ and all the logarithmic ones at $120 \mathrm{~mm}$, but we can expect that the errors for the linear and logarithmic responses should be roughly equally placed above or below 62 and $120 \mathrm{~mm}$, respectively. Hence, if participants were switching there should be roughly as many placements either below the true linear value or above the true logarithmic value as there are between the two values. If, on the other hand, at least some of the participants adopted compromise responses, more than half the responses should be between the linear and logarithmic values.

Table 1 gives the distribution results for the two higher numbers and the two higher markers. The results in the table indicate that at least some participants were adopting compromise placements. Overall, 185 of the 240 placements or $77 \%$ of the placements were between the true linear and logarithmic values rather than the $50 \%$ expected from switching (test of proportion, $p<.0001$ ), and thus the switching hypothesis was disconfirmed.

Six out of 11 participants claimed to have made no use at all of number words for their 10 high marker placements; 6/11 for the 1000 high marker and $8 / 11$ for the $10^{16}$ high marker. Note that the eight participants for the $10^{16}$ high marker condition who did not make use of number words had mean placements at 36, 59,95 and $138 \mathrm{~mm}$ from the left edge (compare Fig. 3 to interpret these results). Thus, the use of number words was not necessary for compressive scaling with the highest marker.

Finally, we fitted power law functions to the average placements. To do this, we subtracted 60 from all the distance placements and fitted the equation (Distance -60$)=$

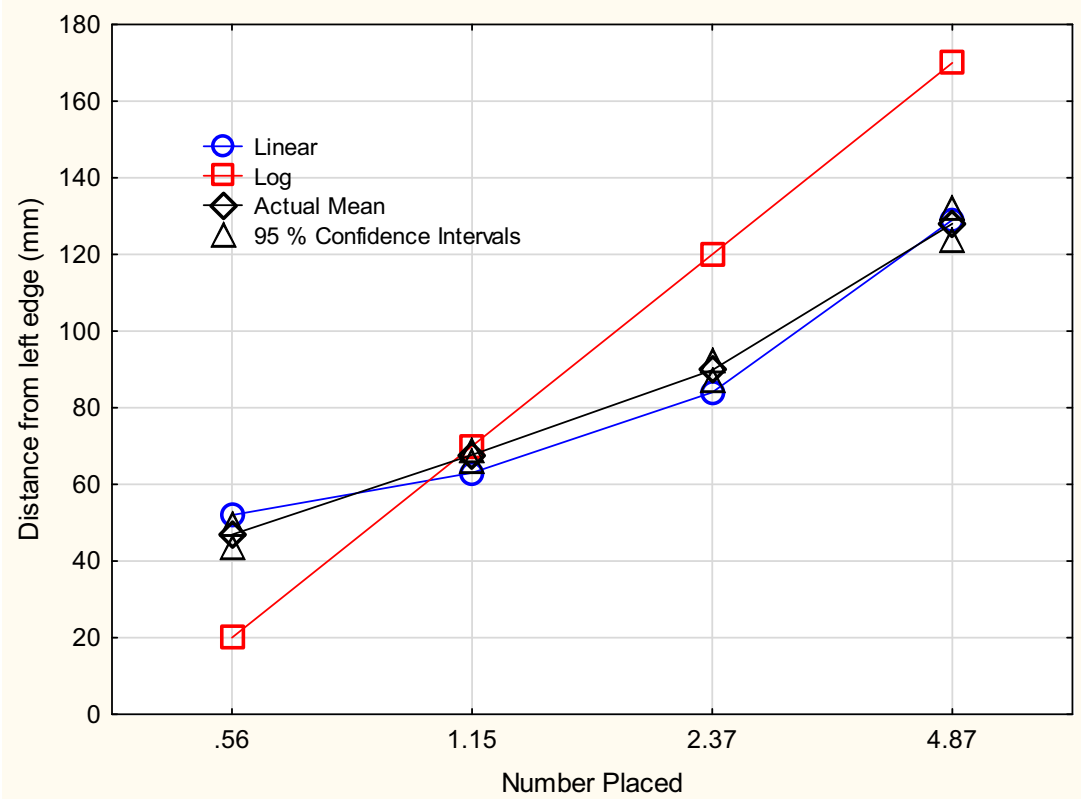

Fig. 1 Actual mean placement value of each number, and 95\% confidence intervals of the mean, when the high marker was 10 in Experiment 1. Also shown are the expected placements if the scaling used was linear and logarithmic 


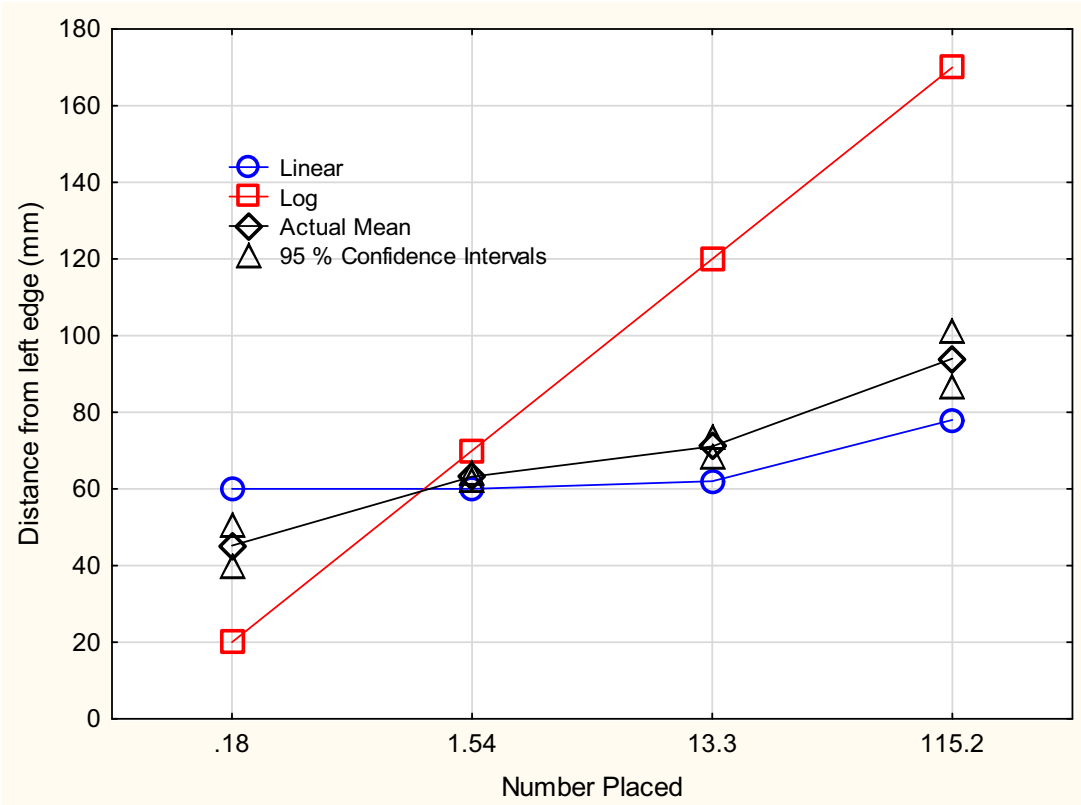

Fig. 2 Actual mean placement value of each number, and 95\% confidence intervals of the mean, when the high marker was 1000 in Experiment 1 . Also shown are the expected placements if the scaling used was linear and logarithmic

$A(\text { Number to place })^{b}$ for the three numbers greater than 1 by taking logarithms and using ordinary least squares regression for each of the three marker conditions. (Note the lowest number was omitted because the average distance was negative and its logarithm does not exist.) The three exponents $(b)$ obtained were 1.51 (High Marker 10; $\left.R^{2}=.98\right), .55$ (High marker $1000 ; R^{2}=1$ ) and .14 (High marker $10^{16} ; R^{2}=.94$ ).

\section{Experiment 2}

This experiment examined the effect of the number distribution (linear or logarithmic) on how a reasonably large sample (14) of numbers was placed on a line. In the main conditions of this experiment (as in Experiment 1) the numbers to be placed on the line were "all in view" together and participants

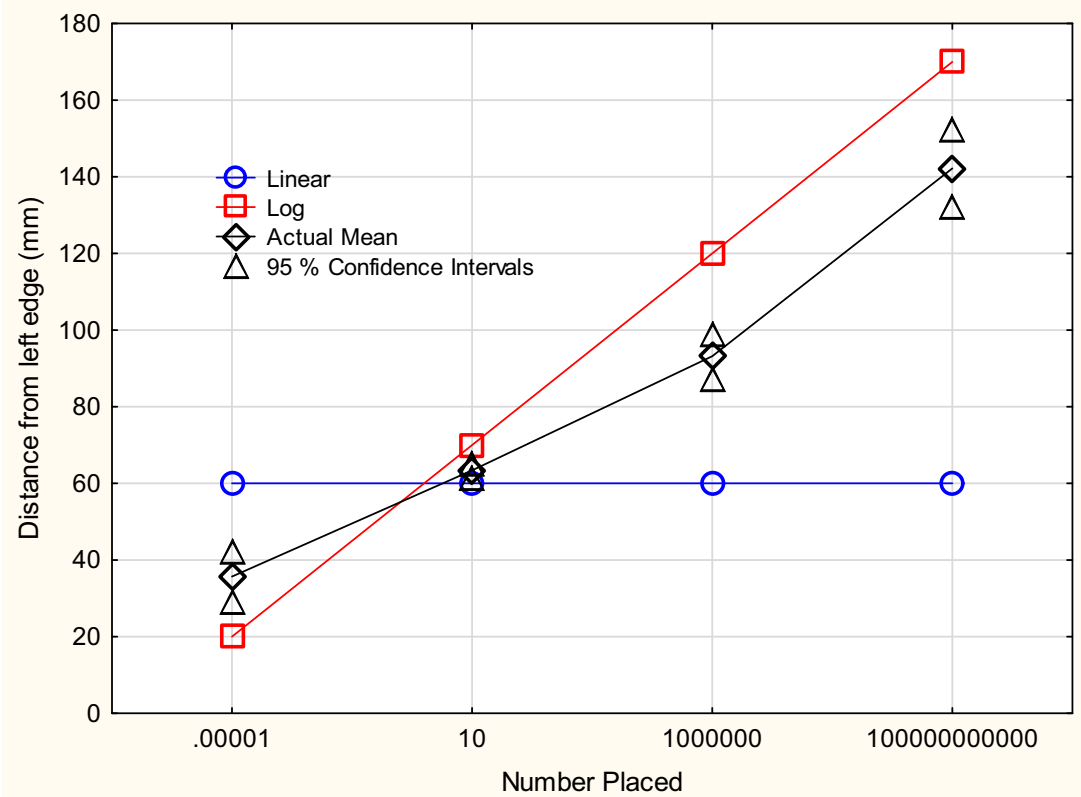

Fig. 3 Actual mean placement value of each number, and 95\% confidence intervals of the mean, when the high marker was 10000000000000000 in Experiment 1. Also shown are the expected placements if the scaling used was linear and logarithmic 
Table 1 Number of participants (total $\mathrm{n}=60$ for all placements) placing values below or equal to the true linear value (in $\mathrm{mm}$ ), between the two true values, equal or above the true logarithmic value, for the two higher numbers on the 1000 and $10^{16}$ high marker lines of Experiment 1

\begin{tabular}{|c|c|c|c|c|c|}
\hline & & \multicolumn{2}{|c|}{ True linear } & \multicolumn{2}{|c|}{ True logarithmic } \\
\hline \multicolumn{6}{|l|}{$10^{16}$ high marker } \\
\hline \multicolumn{6}{|l|}{ Number to place: $10^{6}$} \\
\hline Placing at: & $<60$ & $=60$ & $60<$ placement $<120$ & $=120$ & $>120$ \\
\hline No. of participants: & 0 & 1 & 49 & 1 & 9 \\
\hline \multicolumn{6}{|l|}{ Number to place: $10^{11}$} \\
\hline Placing at: & $<60$ & $=60$ & $60<$ placement $<170$ & $=170$ & $>170$ \\
\hline No. of participants: & 0 & 0 & 43 & 0 & 17 \\
\hline \multicolumn{6}{|l|}{1000 high marker } \\
\hline \multicolumn{6}{|l|}{ Number to place: 13.3} \\
\hline Placing at: & $<62$ & $=62$ & $62<$ placement $<120$ & $=120$ & $>120$ \\
\hline No. of participants: & 1 & 6 & 53 & 0 & 0 \\
\hline \multicolumn{6}{|l|}{ Number to place: 115.2} \\
\hline Placing at: & $<78$ & $=78$ & $78<$ placement $<170$ & $=170$ & $>170$ \\
\hline No. of participants: & 16 & 3 & 40 & 0 & 1 \\
\hline
\end{tabular}

could see all their placements as they went along. This procedure was expected to increase participants' knowledge of what the distribution actually was. In addition, a small sample placed the logarithmically distributed numbers in a "one-ata-time condition". One would not expect distribution effects to be so pronounced in this situation, but they might still be present to some extent. In the "one-at-a-time" condition participants placed single numbers on newly presented "clean" lines. This condition only used the logarithmically distributed numbers (similar to those in "all in view").

\section{Method}

Details of participant numbers in the different conditions of Experiment 2 are given in Table 2.

\section{All-in-view condition}

Seventy-six participants (21 male; median age 22 years, range 18-50+) completed "all-in-view" questionnaires in which they placed 14 different numbers on a single number line. (Another participant, whose placements were markedly disordered, was excluded.)

All participants were presented the same number line. This line was $245 \mathrm{~mm}$ long and had markers 60 and $220 \mathrm{~mm}$ from its left edge. The lower marker was labelled 1 , and the upper marker 1000. (The line was thus identical to the 1000 high marker condition of Experiment 1).

Below the line was a lettered list of 14 numbers. Participants were asked to indicate the placement of each number on the line. They did these under one of two instruction conditions. In one condition $(n=33)$, they were asked to do so by "writing the letter near the line with a little arrow to show where it best fits on the line. Please place the numbers where you think looks good to you. You can place the numbers anywhere on the line, including outside of the arrows." (Similar instructions were used in Experiment 1 and Kemp, 1991). In the other, perhaps more restrictive, instruction condition $(n=43)$ they were told to write in the letters and use "little arrows to indicate where each number should be located on the line." (Similar instructions were used, for example, by Cohen \& Sarnecka, 2014.)

Each participant was presented with either 14 numbers that were close to linearly distributed (that is equally spaced on a linear scale) or 14 numbers that were logarithmically distributed (equally spaced on a logarithmic scale). The actual numbers presented were:

Linear: $0.5,71,143,214,286,356,430,500,573,643$, 715, 786, 857, 928 .

Logarithmic: $0.9,1.6,2.7,4.4,7.2,11.7,19.2,32.5,52.5$, $84.3,138,224,370,605$.

A single random order was used to assign the numbers to the letters (A to $\mathrm{G}$; J to P). This order was preserved across the two conditions. (For example, L was 71 in the linear and 1.6 in the logarithmic condition.)

In total 39 participants placed linearly distributed and 37 logarithmically distributed numbers. Assignment of the different questionnaires to the participants was random.

\section{One-at-a-time condition}

Fifteen participants (ten female; median age 20 years, range 18-35) were recruited. They received psychology course credit for their participation. Participants were tested individually in a quiet room, with the entire experiment taking less 
Table 2 Numbers of participants in the different conditions of Experiment 2

\begin{tabular}{llll}
\hline Number distribution & Linear & Logarithmic & Total \\
\hline "All-in-view" questionnaires & & & \\
Placed "where seemed good to you" & 17 & 16 & 33 \\
Placed "where ... should be located" & 22 & 21 & 43 \\
Totals & 39 & 37 & 76 \\
"One-at-a-time" condition (all placed & & 15 & 15 \\
"where ... should be located") & & & \\
\hline
\end{tabular}

than 10 min to complete. Participants were told to put the number where they thought it should go on the line below.

Numbers were presented one at a time on a computer screen. Below the number was a single line of length 165 $\mathrm{mm}$. The line featured markers, labelled 0.5 (left) and 1000 (right), at either end. Participants responded by clicking on the bar using a mouse. No feedback was given and each trial was followed quickly by the next.

The numbers to be placed on the 14 trials were the same as those used in the logarithmically distributed condition of Experiment 2, except that all numbers were rounded to the nearest whole number. (e.g. 0.9 became 1.) The numbers were always presented in the same order, which was identical to that used in the "all-in-view" condition.

\section{Results}

First, we performed analysis of variance to see if over all 14 letters there was a significant difference in placement between the two distribution sets and the two instruction conditions for the "all-in-view" results. The main purpose of this analysis was to see if the different instructions had any effect. This analysis of variance showed a significant difference between the two number sets, $F(1,72)=206.7, p<.0001, \mathrm{MS}_{\text {Error }}=$ 151.3. (Note, however, that this difference has no practical importance because the numbers in the two sets were quite different.) More importantly, we found no significant main effect of the two instruction conditions, $F(1,72)=2.79, p=$ .10 , or the interaction of number sets and instruction conditions, $F(1,72)=.04, p=.84$. In consequence of this analysis, the remaining results in this section merge data from the two instruction conditions.

Figures 4 and 5 show key results from the experiment. Figure 4 shows the results of presenting the numbers with equal linear spacing. On average, the participant placed the numbers using close to linear spacing, although with a tendency to undershoot. Figure 5 shows the results obtained with the numbers with equal logarithmic spacing. The results show that on average participants used neither equal linear nor equal logarithmic spacing to place the numbers, although the averages are closer to linear than logarithmic spacing.

Signed deviations from the placements that would be expected from linear scaling were computed for each of the 14 linearly distributed numbers. One-sample t-tests showed significant $(p<.05)$ deviation from zero for seven of the numbers $(0.5,286,500,573,643,786,857)$. The largest of these deviations was $-8.8 \mathrm{~mm}$ (for 786). For the log distributed numbers, signed deviations were calculated both from linear and from logarithmic scaling. For the comparison with logarithmic scaling there were significant $(p<.05)$ deviations from zero (all negative) for all but the lowest number (0.9). The largest deviation was $-64.3 \mathrm{~mm}$ (for 84.3 ). For linear scaling there were significant deviations from zero for all the numbers. These deviations were all positive except for the lowest number. The largest deviation was $20.8 \mathrm{~mm}$ (for 138). These statistical tests simply confirm what is evident in Figs. 4 and 5.

As for Experiment 1, we can ask whether the intermediate finding with the logarithmically distributed numbers reflects a mix of individual participants using either logarithmic or linear scaling or most individuals adopting an intermediate position. Table 3 shows the results for the 13 logarithmically distributed numbers greater than 1 . It is clear that, for the lower numbers particularly, individual participants overwhelmingly adopted a compromise position in placing the numbers higher on the number line than would be expected with linear placement but not as much as would be expected from logarithmically scaled placement. (Note, however, that for the two highest numbers (370 and 605), the distributions are reasonably even about the true linear value, in line with the closeness of placements to true linear values in Fig. 5.) In sum, 399 out of 481 of the placements (83\%) fell between the true linear and true logarithmic values, rather than the $50 \%$ predicted under the switching explanation (test of proportion, $p<$ $.0001)$.

We fitted power functions of the form (Average Distance $60)=A\left(\right.$ Number $^{b}$ to the data from both distributions separately, using the 13 data points with numbers greater than 1 . For the linearly distributed numbers, $b=.96\left(R^{2}=1.00\right)$; for the logarithmically distributed numbers, $b=.54\left(R^{2}=.98\right)$.

\section{“One-at-a-time” condition}

In order to make the "one-at-a-time" results comparable with the main results of Experiment 2, all responses on the number line in this experiment were linearly converted to their equivalent placements on the paper line of the "all-in-view" condition. So, for example, 1000 was converted to $220 \mathrm{~mm}, 1$ to 60 $\mathrm{mm}$. Figure 5 shows the average responses so converted from the "one-at-a-time" condition. Confidence intervals for the mean were quite varied (ranging from 59.9-64.0 for placing 1 , to $74.2-115.9$ for placing 138), but generally they were wider than for the "all-in-view" condition. The figure 


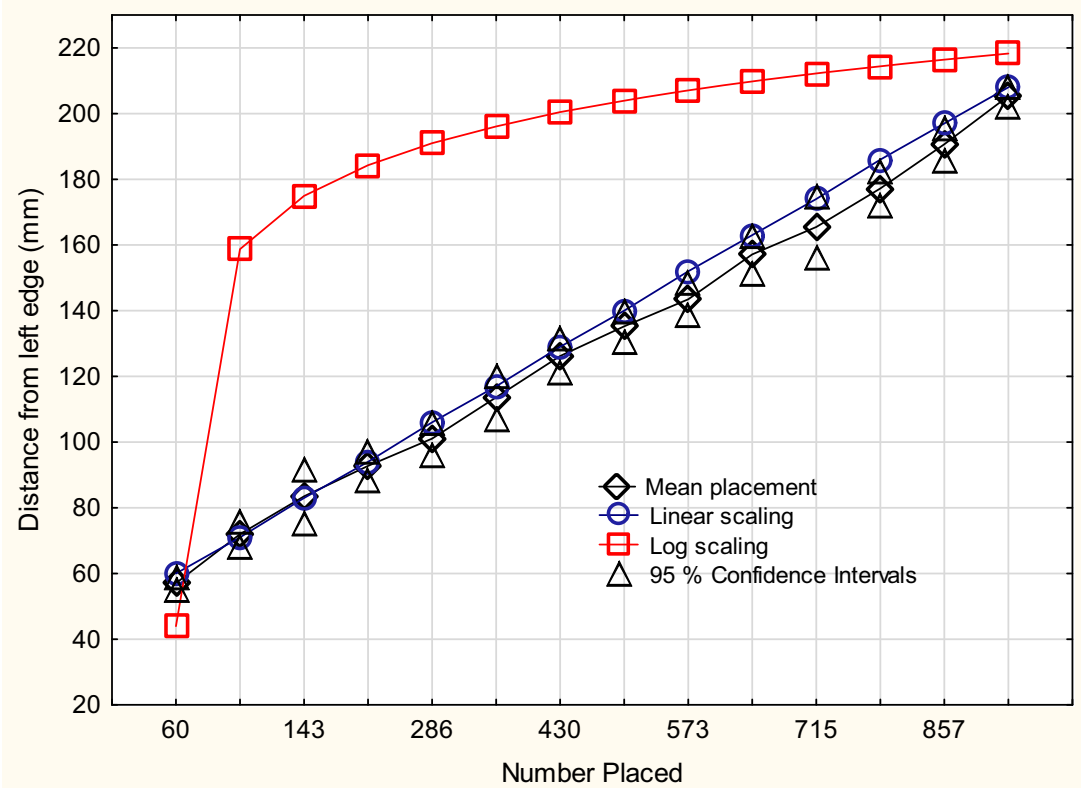

Fig. 4 Mean placement on the line of the linearly distributed numbers, equal linear spacing, and the expected placement from equal logarithmic and $95 \%$ confidence intervals of the mean, as a function of the numbers to spacing (the top curve) be placed in Experiment 2. Also shown are the expected placements from

indicates that the "one-at-a-time" placements were closer to linear than those of the main results, but there is also some evidence of compression. Averaging over the 14 numbers gives a mean placement for the logarithmically distributed numbers of the "all-in-view" condition of $89.2 \mathrm{~mm}$ and the average for the "one-at-a-time" condition was $81.6 \mathrm{~mm}$. A $t$ - test (using participants as the random factor) showed the difference to be significant, $t(50)=2.08, p=.04$. Comparisons for the individual numbers presented showed significant $(t-$ test, $p<.05)$ differences between the two modes of viewing and placement for seven of the numbers $(0.9 / 1,4.4,7.2,32.5$, $84.3,370,605)$. A single-sample $t$-test comparing the mean

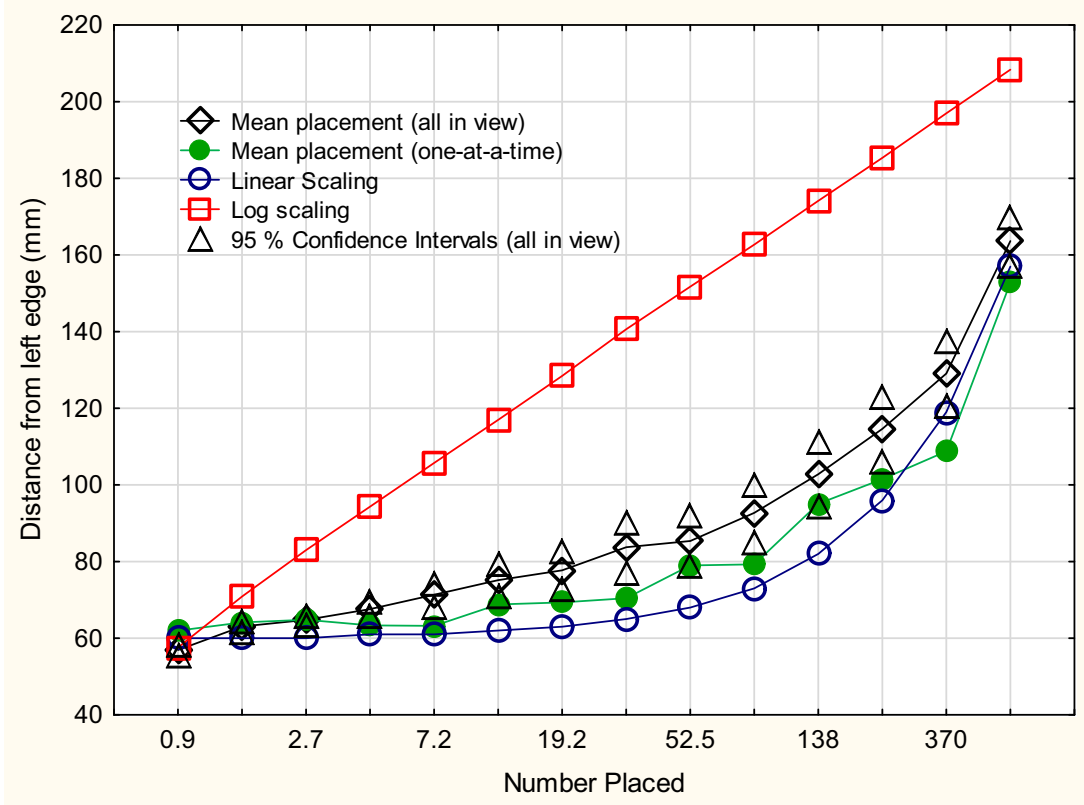

Fig. 5 Mean placement on the line of the logarithmically distributed numbers in Experiment 2 "all-in-view" and "one-at-a-time" conditions. Confidence intervals of the mean $(95 \%)$ are shown for the "all-in-view" condition. Also shown are the expected placements from equal linear spacing (bottom curve) and from equal logarithmic spacing (top straight line) 
Table 3 Number of participants (total $\mathrm{n}=37$ for all placements) placing values below or equal to the true linear value, between the two true values, equal or above to true logarithmic value, for the higher 13 numbers to be placed in the logarithmically distributed condition of Experiment 2

\begin{tabular}{llcccc}
\hline Number to place & \multicolumn{3}{c}{$\begin{array}{c}\text { True linear } \\
=\end{array}$} & between & $\begin{array}{c}\text { True logarithmic } \\
=\end{array}$ \\
\hline 1.6 & 1 & 4 & 31 & 0 & 0 \\
2.7 & 1 & 1 & 35 & 0 & 0 \\
4.4 & 0 & 0 & 37 & 0 & 0 \\
7.2 & 0 & 1 & 36 & 0 & 0 \\
11.7 & 1 & 2 & 34 & 0 & 0 \\
19.2 & 2 & 1 & 34 & 0 & 0 \\
32.5 & 2 & 0 & 34 & 0 & 1 \\
52.5 & 5 & 1 & 31 & 0 & 0 \\
84.3 & 5 & 1 & 31 & 0 & 0 \\
138 & 9 & 0 & 28 & 0 & 0 \\
228 & 7 & 1 & 28 & 0 & 0 \\
370 & 18 & 0 & 19 & 0 & 0 \\
605 & 15 & 0 & 21 & 1 & 0 \\
\hline
\end{tabular}

placement for the "one-at-a-time" condition with the mean expected linear placement $(77.6 \mathrm{~mm})$ also showed a significant difference, $t(15)=2.30, p=.04$.

\section{Discussion}

Both Experiment 1 and Experiment 2 produced results that were predicted from Parducci's (1965) range-frequency theory. Experiment 1 found that using a very large number range leads to more number compression than a small $(1: 10)$ or moderate (1:1000) range, as predicted from range-frequency theory. One might object that the largest range of numbers in this study is completely outside the experience of the participants, but the ratio of the smallest to the largest numbers $\left(10^{-4}: 10^{11}\right.$ or $\left.1: 10^{15}\right)$ to be placed was equal to the range of sound intensities humans encounter. Thus, the range presented was not outside the range of our experience of sensory qualities.

As noted in the Introduction, Landy et al. (2013, 2017) previously reported range effects. A principal source of these effects in their research seems to have been the use of number words, but this does not seem to be the explanation of our results. Most participants who were asked about their use of number words reported not using them. Moreover, the highest high marker stimuli we used were not easy to verbalise. Ten quadrillion $\left(10^{16}\right)$ and a hundred billion $\left(10^{11}\right)$ are not commonly used words. Moreover, our omission of commas in the number presentations was chosen to direct participants' attention to the length of the number or number of zeros rather than to a verbal description. In consequence, our range effects probably came about through a different context-producing process than those range effects found by Landy et al. (2013, 2017).

Experiment 2 showed compression to be affected by the distribution of the numbers, again as predicted from rangefrequency theory. A linear (uniform) number distribution there gave rise to near linear placement of the numbers, whereas a logarithmic distribution gave rise to more compressive placement. Somewhat different forms of the instructions did not have a significant effect on this result. The more usual experimental paradigm of presenting numbers to be placed one at a time on new, clean lines did lead to a reduction in the strength of the frequency distribution effect. Of course, in the one-at-a-time paradigm there was no opportunity for participants to see the actual frequency distribution at a glance and a reduced opportunity for learning it as they placed successive numbers.

The experiments were designed so as to enable a logarithmic compressive function to appear in at least some experimental conditions. We used 1 as a lower marker in both studies and equal logarithmic placement in all conditions of Experiment 1 and the logarithmically distributed condition of Experiment 2. Yet, despite such nudges towards the use of a logarithmic function, no experimental condition actually produced one. This is evident from Figs. 1, 2, 3, 4 and 5. Instead, the placement means tended to follow functions that were intermediate between linear and logarithmic.

An obvious explanation of the average intermediate functions would be that some participants might use a linear function and others a logarithmic function, and the number of individuals doing each might vary with the experimental conditions and with the actual number to be placed. The result of such switching would be average data that were intermediate. However, the analyses of individual responses for Experiments 1 and 2 indicate this is not the correct explanation as the distribution of individual responses indicated intermediate placement rather than bimodal linear and logarithmic placement.

The finding that individual participants place numbers in a way that is intermediate between linear and logarithmic placement is in line with previous research indicating segmentation in placement on number lines (e.g. Landy et al., 2013; Moeller et al., 2009). It also resembles a finding from Grace et al. (2018) that participants often responded in a way that mixed ratios and differences in comparing stimulus pairs, rather than opting for one or the other.

Thus, our experiments indicate that where compressive functions arise, the compressive function is not logarithmic. So, what is it? Our studies fall short of answering this question, but some tentative suggestions can be made. It is possible that the compressive function follows a power law, but, if so, our results show that it follows a power law whose exponent changes with both the range and distribution of the numbers to be placed. This is possible and, in the case of stimulus range, in line with previous research on power functions in 
psychology generally (Kowal, 1993; Poulton, 1968; Teghtsoonian, 1973). On the other hand, it is not theoretically very satisfying.

Another possibility that is consistent with the data presented here, is that, following Parducci (1965), placements are heavily influenced by the range and distribution of the presented numbers. This influence may be such as to overwhelm any simple mathematical function. However, a detailed explanation of how this might occur requires not only more empirical work but also a coherent explanation of how rangefrequency theory should be developed in order to accommodate continuous rather than categorical variables. Larger scale versions of the "one-at-a-time" condition of Experiment 2 might help resolve this issue (cf. Cicchini et al., 2014; Haubensak, 1992).

The effects of range and frequency found here resemble the effects of number words (e.g. Landy et al., 2013), training effects (Huber et al., 2014), number-line midpoint practice (Barth \& Paladino, 2010), cultural effects (Göbel et al., 2011), and boundary provision (Cohen \& Sarnecka, 2014), in that they indicate the importance of context in how people assign numbers to number lines. Given all these different effects, it is difficult to imagine that there is any one basic number or magnitude representation.

To summarise, the experiments presented here show that both the range (provided the range is great enough) and the distribution of the numbers (provided there are enough numbers) affect the degree of compression when numbers are placed on number lines. The compression is not simply described by a logarithmic function, but it is not yet clear what the compressive function is. Indeed, it is not clear that there is a single compressive function.

Author Notes Georgina Carvell died suddenly and unexpectedly before this paper was accepted. The remaining authors dedicate this paper to her memory, with fond memories of her intellect and kindness.

The research was supported by the Marsden Fund Council from Government funding, administered by the Royal Society of New Zealand.

Open Practices Statement The data for both experiments are publicly available from the Open Science Framework under Simon Kemp's Quick Files (NumberlineE1data.xlsx; NumberlineE2data.xlsx). The experiments were not preregistered.

\section{Appendix. Shown below is the layout used for the Experiment 1 higher marker $=1000$ page}

$\begin{array}{rrrr}4 & 4 & 1000 & 1\end{array}$

Above is a number line. Below is a list of four numbers. Please indicate how you would like to place each number on the line by writing it near the line with a little arrow to show where it best fits in on the line. Please place the numbers where you think looks good to you. You can place the numbers anywhere on the line, including outside of the arrows.

\section{3 \\ 0.18 \\ 1.54 \\ 115.5}

\section{References}

Barth, H. C., \& Paladino, A. M. (2010). The development of numerical estimation: Evidence against a representational shift. Developmental Science, 14, 125-135. https://doi.org/10.1111/j.1467-7687.2010. 00962.x

Cicchini, G. M., Anobile, G., \& Burr, D. C. (2014). Compressive mapping of number to space reflects dynamic encoding mechanisms, not static logarithmic transform. PNAS, 111, 7867-7872. https://doi.org/ 10.1073/pnas. 1402785111

Cohen, D. J., \& Blanc-Goldhammer, D. (2011). Numerical bias in bounded and unbounded number line tasks. Psychonomic Bulletin \& Review, 18, 331-338. https://doi.org/10.3758/s13423-011-0059-z

Cohen, D. J., \& Sarnecka, B. (2014). Children's number-line estimation shows development of measurement skills (not number representations). Developmental Psychology, 50, 1640-1652. https://doi.org/ 10.1037/a0035901

De Hevia, M-D, \& Spelke, E. S. (2009). Spontaneous mapping of number and space in adults and young children. Cognition, 110, 198-207. https://doi.org/10.1016/j.cognition.2008.11.003

Dehaene S. (1997) The number sense: How the mind creates mathematics. Oxford: Oxford University Press.

Dehaene, S., Izard, V., Spelke, E., \& Pica, P. (2008). Log or linear? Distinct intuitions of the number scale in Western and Amazonian indigene cultures. Science, 320, 1217-1220. https://doi.org/10. 1126/science. 1156540

Didino, D., Pinheiro-Chagas, P., Wood, G., \& Knops, A. (2019). Response: Commentary: The developmental trajectory of the operational momentum effect. Frontiers in Psychology, 10, 160. https:// doi.org/10.3389/fpsyg.2019.00160

Dotan, D., \& Dehaene, S. (2016). On the origins of logarithmic numberto-position mapping. Psychological Review, 123, 637-666. https:// doi.org/10.1037/rev0000038

Droit-Volet, S., Clément, A., \& Fayol, M. (2008). Time, number and length: Similarities and differences in discrimination in adults and 
children. Quarterly Journal of Experimental Psychology, 61, 18271846. https://doi.org/10.1080/17470210701743643

Galanter, E. (1962a). New directions in psychology. New York: Holt.

Galanter, E. (1962b). The direct measurement of utility and subjective probability. American Journal of Psychology, 75, 208-220. https:// doi.org/10.2307/1419604

Göbel, S. M., Shaki, S., \& Fischer, M. H. (2011). Cultural effects on the mental number line. Journal of Cross-Cultural Psychology, 42, 541-542. https://doi.org/10.1177/0022022111406019

Grace, R. C., Morton, N. J., Ward, M. D., Wilson, A. J., \& Kemp, S. (2018). Ratios and differences in perceptual comparison: A reexamination of Torgerson's conjecture. Journal of Mathematical Psychology, 85, 62-75. https://doi.org/10.1016/j.jmp.2018.07.004

Grice, M., Kemp, S., Morton, N. J, \& Grace, R. C. (2020). Monotonicity, convexity, continuity, and isomorphism: The psychological scaffolding of arithmetic. PsyArXiv. https://doi.org/10.31234/osf.io/ myq4n

Haubensak, G. (1992). The consistency model: A process model for absolute judgments. Journal of Experimental Psychology: Human Perception and Performance, 18, 303-309. https://doi.org/10.1037/ 0096-1523.18.1.303

Huber, S., Moeller, K., \& Nuerk, H-C. (2014). Dissociating number line estimations for underlying numerical representations. Quarterly Journal of Experimental Psychology, 67, 991-1003. https://doi. org/10.1080/17470218.2013.838974

Izard, V., \& Dehaene, S. (2008). Calibrating the mental number line. Cognition, 106, 1221-1247. https://doi.org/10.1016/j.cognition. 2007.06.004.

Kemp, S. (1991). Magnitude estimation of the utility of public goods. Journal of Applied Psychology, 76, 533-540. https://doi.org/10. 1037/0021-9010.76.4.533

Kowal, K. H. (1993). The range effect as a function of stimulus set, presence of a standard, and modulus. Perception and Psychophysics, 54, 555-561. https://doi.org/10.3758/BF03211777

Landy, D. Silbert, N., \& Goldin, A. (2013). Estimating large numbers. Cognitive Science, 37, 775-799. https://doi.org/10.1111/cogs. 12028.

Landy, D., Charlesworth, A., \& Ottmar, E. (2017). Categories of large numbers in line estimation. Cognitive Science, 41, 326-353. https:// doi.org/10.1111/cogs.12342

Moeller, K., Pixner, S., Kaufmann, L., \& Nuerk, H-C. (2009). Children's early mental number line: Logarithmic or decomposed linear? Journal of Experimental Child Psychology, 103, 503-515. https:// doi.org/10.1016/j.jecp.2009.02.06

Moore, B. J. (2004). An introduction to the psychology of hearing, 5th Edition. New York: Academic Press.
Núñez, R. E. (2011). No innate number-line in the human brain. Journal of Cross-Cultural Psychology, 42, 651-668. https://doi.org/10. 1177/0022022111406097

Parducci, A. (1965). Category judgment: A range-frequency model. Psychological Review, 72, 407-418. https://doi.org/10.1037/ h0022602

Parducci, A. (1974). Contextual effects: A range-frequency analysis. In E. C. Carterette \& M. P. Freidman (Eds.), Handbook of perception: Vol.II (pp. 127-172). New York: Academic Press.

Parducci, A., \& Wedell, D.H. (1986). The category effect with rating scales: Number of categories, number of stimuli, and method of presentation, Journal of Experimental Psychology: Human Perception and Performance, 12, 496-516. https://doi.org/10. 1037/0096-1523.12.4.496

Poulton, E. C. (1968). The new psychophysics: six models for magnitude estimation. Psychological Bulletin, 69, 1-19. https://doi.org/10. 1037/h0025267

Siegler, R. S. (2009). Improving the numerical understanding of children from low-income families. Child Development Perspectives, 3, 118-124. https://doi.org/10.1111/j.1750-8606.2009.00090.x

Siegler, R. S., \& Booth, J. L. (2004) Development of numerical estimation in young children. Child Development, 75, 428-444. https://doi. org/10.1111/j.1467-8624.2004.00684.x

Slusser, E., \& Barth, H. (2017). Intuitive proportion judgment in numberline estimation: Converging evidence from multiple tasks. Journal of Experimental Child Psychology, 162, 181-198. https://doi.org/ 10.1016/j.jcep.2017.04.010

Stevens, S. S. (1961). To honor Fechner and repeal his law: A power function, not a log function, describes the operating characteristic of a sensory system. Science, 133(3446), 80-86. https://doi.org/10. 1126/science.133.3446.80

Stevens, S. S. (1975). Psychophysics: Introduction to its perceptual, neural, and social prospects. New York: John Wiley.

Teghtsoonian, R. (1973). Range effects in psychophysical scaling and a revision of Stevens' Law. American Journal of Psychology, 86, 3 27. https://doi.org/10.2307/1421845

Zax, A., Slusser, E., \& Barth, H. (2019). Spontaneous partitioning and proportion estimation in children's numerical judgments. Journal of Experimental Child Psychology, 185, 71-94. https://doi.org/10. 1016/j.jecp.2019.04.004

Publisher's note Springer Nature remains neutral with regard to jurisdictional claims in published maps and institutional affiliations. 